\title{
Emotional Cascade Model and Deep Learning
}

\author{
Carlos Pelta \\ Dept. of Experimental Psychology \\ Cognitive Processes and Speech Therapy \\ UCM, Madrid, Spain
}

\begin{abstract}
Emotional Cascade Model proposes that the emotional and behavioral dysregulation of individuals with Borderline Personality Disorder can be understood through emotional cascades. Emotional cascades are vicious cycles of intense rumination and negative affect that may induce aversive emotional states that generate abnormal behaviors to reduce the effect of intense rumination. Borderline Personality Disorder is a psychiatric disorder whose main symptoms to diagnose it are mood instability and impulsivity. This disorder often involves risky behaviors such as non-suicidal self-injury or substance abuse. Recently, Selby and collaborators have proved that the Emotional Cascade Model has a high explanatory and diagnostic capacity using Temporal Bayesian Networks. Taking into consideration the meta-analytic study developed by Richman et al., in this article it has been designed a deep learning model, based on cascading artificial neural networks, following the correlations established for the Emotional Cascade Model. It has been confirmed with accuracy estimates reaching up to $99 \%$, the predictive power of this model relative to the various types of rumination that influence some of the basic classes of symptoms of Borderline Personality Disorder.
\end{abstract}

Keywords-Emotional cascade model; borderline personality disorder; rumination; deep learning; cascade-correlation algorithm

\section{INTRODUCTION}

Borderline Personality Disorder (BPD) is a psychiatric disorder characterized by symptoms such as affect instability, impulsivity, self-harm and identity disturbances [1]. Such syndrome causes behaviors as suicidality [2], non-suicidal self- injury (NSSI) [3], chronic pain [4], substance abuse [5], binge-eating [6], and gender violence [7]. The pioneering and perhaps most complete theory of BPD has been that of Linehan [8]. It is a biological, psychological and social theory claiming that individuals with BPD develop extreme emotional vulnerability.

Selby, Anestis and Joiner [9] propose in their Emotional Cascade Model (ECM) that the cycle of affect and rumination (the emotional cascade) leads to dysregulated behavior that functions to distract attention from this negative internal experience. Rumination is typical in people with BPD and predicts dysregulated behavior [10]. It is a form of repetitive thinking in which individuals focus their attention on emotionally relevant stimuli [11,12]. Rumination usually magnifies negative affect. According to the ECM, the reciprocal relationship between negative affect and ruminative processes results in an "emotional cascade", which is initiated by an event that elicits an emotion. This event causes the individual to ruminate intensely, increasing the intensity of the emotion. As the intensity of the emotion increases it is more difficult to escape from the emotional experience paying full attention to the emotional stimulus. Then, it is generated a positive feedback loop between rumination and negative affect. There is a synergistic [13] effect between rumination and negative emotion predicting impulsive behaviors typical of BPD and forming emotional cascades [14]. Emotional cascades create a complex disorder that may be involved in the emergence of BPD. Emergence is the phenomenon by which a complex system arises from the interaction of its component network [15]. Broadly speaking, there is bidirectionality between emotional cascades and symptoms of BPD.

There are various types of rumination. The most common in BPD patients are pain rumination [16], depressive rumination [17,18] and anger rumination [19]. Anger rumination is repetitive thinking about anger experiences and has maladaptive outcomes such as physiological arousal or aggressive behavior. Stress and anxiety rumination can also appear [20,21]. Nolen-Hoeksema conceptualizes the depressive rumination as repetitively focusing on the fact that one is depressed and the possible causes and consequences of the depressive symptoms [22].

Richman et al. [23] have performed a meta-analysis to assess how the four main categories of BPD symptoms (affect-instability, relationship issues, self-harm/impulsivity, and identity disturbances) correlate with the five types of rumination mentioned above (pain rumination, depressive rumination, anger rumination, anxious and stress rumination). This meta-analysis of the correlations extracted between the data provided by 13 studies has supplied us with the basis for designing a cascading artificial neural network capable of simulating the ECM for BPD. Cascade-correlation (CasCor) [24] is an algorithm starting with a minimal network architecture, to which hidden nodes are added as required, forming feature detectors within the network. It is an algorithm especially suitable for modeling developmental cognitive cascades as Shultz does in [25] or processes such as representational redescription [26, 27]. The author of this article has also used it to model the psychological phenomenon of catastrophic worry [28] or the interaction between cytokines appearing together with lymphopenia in COVID-19 disease [29].

Deep learning uses multiple layers with non-linear processing units to extract and transform features. Each layer uses the output of the previous layer as input. Cascadecorrelation algorithm is a pioneering algorithm in this type of machine learning, since it adds new layers as new neurons are added, in a constructive process that avoids the disadvantage 
of having to predeterminate the structure of an artificial neural network from the start [30] and adapts very adequately to the modeling of any cascade phenomena by means of "cascades" of layers.

In this article is demonstrated, using deep learning techniques, how it is possible to computationally model with an exceedingly small error the interaction between the network elements that intervene in the emotional cascades typical of patients with BPD. Thus, it has been reinforced the predictive value obtained by Selby and collaborators [31] about their model of emotional cascades of psychological disorders and it has been reaffirmed, using the technique of artificial neural networks, the value of their model as a source of explanation for these disorders. Using a different computational technique, it has been improved the accuracy in the validation of the model-reaching $99 \%$.

\section{LITERATURE REVIEW}

Computational psychiatry [32] is a recent field that addresses a phenomenon as complex as mental illness using computational tools. We can distinguish between biophysical models (for instance, in [33] has been studied the role of dopamine in the central cortex of patients with schizophrenia), reinforcement learning models (for example, in [34] has been investigated the relation between reinforcement learning and the diagnosis of Alzheimer Disease), connectionist models (the first major paper on a neural network model of psychopathology [35] simulated the processing of the information on schizophrenia and in the 1990s came the first anthologies of articles [36,37]) and Bayesian network models.

Selby et al. [31] have proposed a Temporal Bayesian Network (TBN) model to examine the ECM in a sample of adolescents and young adults who actively self-injure and including those with BPD.

TBN is an extension of the Bayesian technique incorporating time dependence [38]. A TBN model consists of several connected clusters of nodes, one cluster corresponding to one-time step. The clusters of nodes at different time steps are interconnected in the same way within each cluster. The connections among the clusters of nodes correspond to the time dependence of the variables, updating one's knowledge of other variables by calculating the posterior probability distribution over the remaining variables given the obtained evidence. Four core features of the ECM were modeled and used in model prediction evaluation: (a) rumination and negative emotion generate positive feedback on each other, (b) they generate positive feedback on themselves, (c) these positive feedbacks provoke dysregulated behavior, and (d) dysregulated behavior then reduces rumination and negative emotion. Time invariant variables for psychiatric diagnosis were specified. These variables were BPD, Major Depressive Disorder (MDD), and Posttraumatic Stress Disorder (PSD) and they were used in determining model predictive accuracy of BPD diagnosis, state rumination, negative emotion, and dysregulated behaviors. The predictive accuracy was then evaluated based on real-world data and using the $k$-fold cross-validation method [39] on interim models. Several interim models were initially built. For each of the models, a model structure was first generated using causally connected nodes corresponding to the momentary negative emotion, rumination, and dysregulated behavior, measured at five-time steps during each day, and including nodes corresponding to the diagnostic statuses of the participants. TBN analysis suggested that the ECM predicted BPD diagnosis (with accuracy estimates around 90\%) and momentary prediction of rumination, negative emotion, and dysregulated behaviors with accuracy estimates above $80 \%$ and reaching up to $100 \%$, depending on the level of momentary prediction selected.

\section{MATERIALS AND METHOD}

\section{A. Database}

The database used the correlations between BPD symptoms and types of rumination obtained by Richman et al. [23] selecting the results provided by 13 articles. Following to [23], when correlation values were not available, correlation values were calculated from reported univariate t-statistics, or p-values. Correlations were classified as small (0.1-0.3), medium (0.3-0.5), and large (0.5-1). To assess homogeneity of the correlation sizes across studies for each rumination domain, the Cochran Q-statistic was used. In all cases, significant at $\mathrm{p}<.001$ and $95 \%$ CI. For $N=28,165$, Table I shows the types of rumination correlation with BPD symptoms that have been used as the parameters for adjusting the weights of the connections between the units of the neural network.

Besides, Table II shows the BPD symptoms correlation with rumination for the same sample. Also, these correlations have served for adjusting the weights of the artificial neural network.

TABLE I. TYPES OF RUMINATION CORRELATION WITH BPD SYMPTOMS

\begin{tabular}{|l|l|}
\hline Types of rumination & Overall BPD symptoms correlation \\
\hline Overall rumination & 0.41 \\
\hline Pain rumination & 0.68 \\
\hline Anger rumination & 0.46 \\
\hline Depressive rumination & 0.39 \\
\hline Stress rumination & 0.35 \\
\hline Anxious rumination & 0.28 \\
\hline
\end{tabular}

TABLE II. BPD SYMPTOMS CORRELATION WITH RUMINATION

\begin{tabular}{|l|l|}
\hline BPD symptoms & Overall rumination \\
\hline Overall symptoms & 0.43 \\
\hline Affective instability & 0.42 \\
\hline Unstable relationship & 0.39 \\
\hline Identity disturbance & 0.37 \\
\hline Self-harm/impulsivity & 0.25 \\
\hline
\end{tabular}




\section{B. Design of the Artificial Neural Network}

It has been used MemBrain (version V03.08.01.00) software, created by Jetter [40], for the design of the artificial neural network. Cascade-correlation algorithm seems adequate to simulate the ECM and, therefore, the positive feedback between types of rumination and the main BPD symptoms. $\mathrm{BPD}$ is an emerging phenomenon that allows explaining, through the spread of emotional cascades, why unregulated behaviors are succeeding each other. Subjects respond more and more negatively towards these behaviors and their cognitions are distorted in a process that Selby [41] calls "expectancy validation" and that adds more suffering to the subjects.

The cascade-correlation architecture is a multi-layer supervised connectionist learning system. The most interesting difference between it and other multilayer systems is that in a cascading artificial neural network the number of hidden units is not predetermined. Instead, these units are added as necessary and achieve a progressive reduction in error. The network starts with only specified inputs and outputs. Neurons are typically trained off-line, and a pool of new hidden neurons or candidates is created. The best of these candidates is situated in a separate layer and connected to the output neurons. If a candidate correlates positively with the error at a given neuron, it will develop a negative weight to compensate for that error, while if the correlation is negative, the weight will be positive. The weights on the connections to the new hidden neurons are frozen and the input-output and hiddenoutput connections are re-trained to readjust overall performance. This process is repeated until the total error at the output has converged or there has been a lack of significant improvement in error (stagnation) over a period of epochs (patience).

The learning algorithm has been cascade-correlation using backpropagation and target net error has been 0.01 . The weights of the connections between the neurons have been initially randomized until adjusting to the values indicated in Table I and Table II. A symmetric logistic activation function has been used with values between -0.5 and 0.5 and an error threshold of 0.4. We have established as parameters, the learning rate $(\eta)$, the maximum growth rate $(\mu)$ which limits the size of any change in weights performed after each presentation of the training set, the weight decay $(\gamma)$ which ensures that the weights do not grow excessively, the patience percentage calculated from the change in error required over a period to continue training, the patience period or the period over which the change in error is measured through epochs (or lesson runs), and the activation function offset. When the maximum number of epochs has been reached or no progress has been made in the training of the candidate neurons, the best candidate is placed into the network and the output layer is retrained. The algorithm cycles through installing hidden nodes. In the following Table III, we indicate the values for output layer training parameters.

It has been designed the artificial neural network by introducing 11 units, of which the input neuron has been overall rumination (O-RUM), in a first layer, 5 neurons have been placed referring to the 5 types of rumination (P-RUM, ARUM, D-RUM, S-RUM, AX-RUM, respectively) and the candidate neurons have been the units corresponding to the four main types of BPD symptoms (AI, UR, ID and SH), to end in the output O-BPD that corresponds to the overall BPD.

The maximum number of candidates has been 8 and the size of the set of candidates has been 4 . For the training candidates, the correlation stable tolerance has been $5 \%$.

The structure of the network can be seen in the Fig. 1 and the training parameters of the candidates can be seen in the Fig. 2.

TABLE III. OUtPut LAYER TRAINING PARAMETERS

\begin{tabular}{|l|l|}
\hline Parameter & Output value \\
\hline$\eta$ & 0.4 \\
\hline$\mu$ & 1.5 \\
\hline$\gamma$ & 0.0 \\
\hline Patience percentage & $1 \%$ \\
\hline Patience period & 300 \\
\hline Activation function offset & 0.1 \\
\hline & \\
\hline & \\
\hline & \\
\hline & \\
\hline
\end{tabular}

Fig. 1. Structure of the ANN (All the Neurons are Activated in Red and the Connections in Red Express Positive Weights).

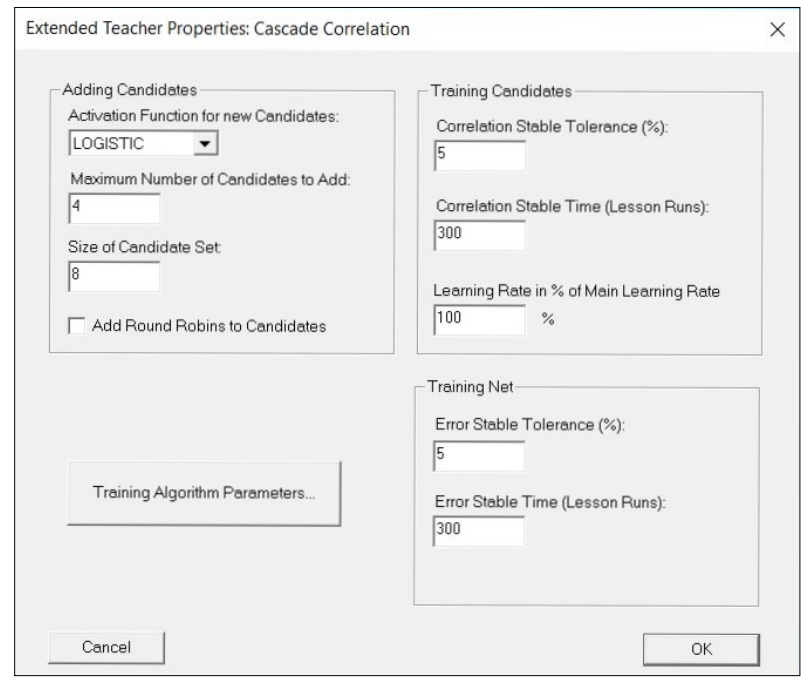

Fig. 2. Training Parameters of the Candidates in the Net. 


\section{RESUlT AND DisCUSSION}

The learning rule has been applied to minimize the Mean Squared Error (MSE), which is calculated with the average difference between the observations and the expected values, obtaining the result with the iterations produced with the help of the learning algorithm. As Fig. 3 of the Net Error Graph shows, the Net Error has been 0.00991199644037891.

This result proves that the correlations established by the meta-analytic study [23] between types of rumination and overall rumination and the main classes of BPD symptoms and overall BPD allow designing a computational simulation using an artificial neural network in cascade that adjusts in a remarkably high degree to these correlations. By the way, it has been improved the precision in the validation of the ECM by means of a different computational technique. Selby and colleagues used a Temporal Bayesian Network and, in this article, has been employed a cascading neural network. Using this computational tool, it has been reached a validation of the ECM of almost $100 \%$.

The fundamental merit of the ECM is to capture very well the inherently dynamic and cascading nature of the BPD as it is fed by rumination and the negative affect generated. In other perspectives, BPD is understood as a hardwired disorder activated by emotions. In the ECM, the BPD symptoms are increased by an intense rumination process that, in turn, generates negative emotional states. BPD unfolds over time periods far beyond the presence of the emotions that caused the symptoms, and the component processes themselves (called "hysteresis"), a nonlinearity that is typical for complex systems. The symptoms are maintained and affect the emotional state space influenced by rumination and negative emotions. Of course, not all rumination processes are identical, but BPD cascade is amplified by a persistent rumination process.

BPD psychopathological preconditions and effects are accurately simulated by the computational architecture of a cascading artificial neural network, as we have tried to demonstrate in this article.

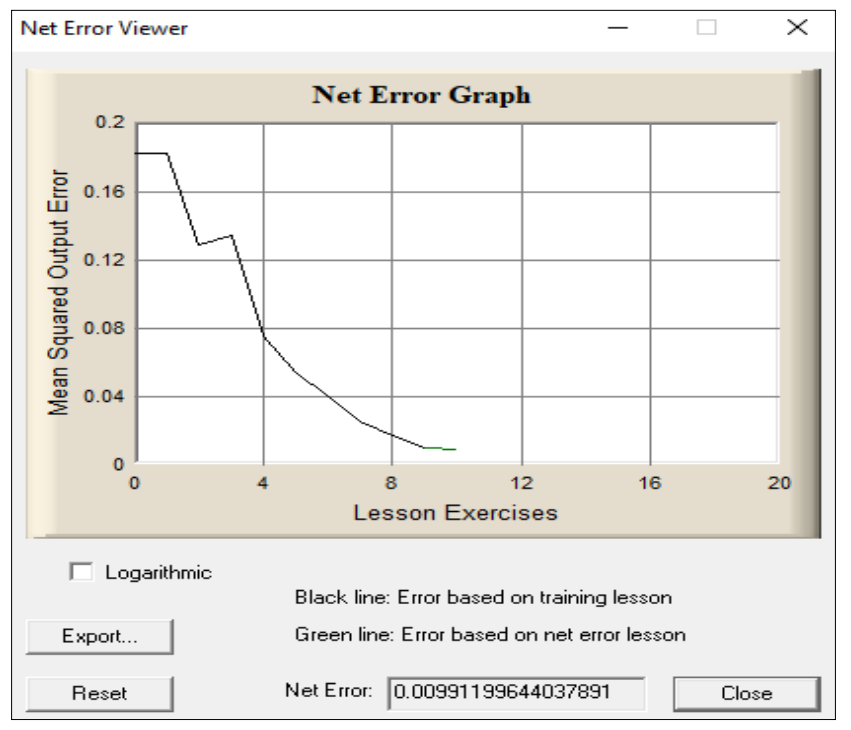

Fig. 3. Net Error.
Really, the cascade-correlation algorithm is suitable for modeling numerous psychopathological processes that present a cascade structure, that is, processes that take place in a staggered fashion and that start a cycle with positive feedback. For instance, disorders such as neurosis or catastrophic pain deserve to be computationally modeled using this kind of artificial neural network. Furthermore, psychological processes involving developmental cascades, that is, processes by which function at one level of behavior affect the organization of competency in later developing domains, admit to being modeled following this methodology and not simply the usual models of structural equations that do not capture the constructive and evolutionary nature of this class of systems.

\section{CONCLUSION}

This article has sought to design a suitable artificial neural network (ANN) for simulating the Emotional Cascade Model (ECM) by Selby and collaborators applied to the borderline personality disorder (BPD). According to ECM, the main BPD symptoms are triggered by an insidious rumination process that generates negative emotional states. These negative emotional states, in turn, intensify the rumination types and the borderline symptoms. The ANN type has been a cascadecorrelation network with backpropagation. Cascadecorrelation architecture is well suited for modeling cascading events. These events are self-amplifying cascades of strong negative emotions. Cascading networks with backpropagation grow in a similar manner to the process of emotional cycles typical of the ECM and have allowed us to validate the correlations between the main symptoms of BPD.

\section{REFERENCES}

[1] A.W. Bateman and R. Krawitz, Borderline personality disorder. Oxford: OUP, 2013.

[2] J.G. Gunderson and M.A. Ridolfi, "Borderline personality disorder: suicidality and self-mutilation", in The clinical science of suicide prevention, H.Hendin and J.J. Mann, Eds., NY: NYAS, 2001, pp. 61-77.

[3] P.A. Hasking, M. Di Simplicio, P.M. McEvoy and C. S. Rees, "Emotional cascade theory and non-suicidal self-injury: the importance of imagery and positive affect”, Cogn Emot, 32, pp. 941-952, 2018.

[4] J. Dersh, R.J. Gatchel, T. Mayer, P. Polatin and O. R. Temple, "Prevalence of psychiatric disorders in patients with chronic disabling occupational spinal disorders, Spine, 31, pp. 1156-1162, 2006.

[5] S. Nolen-Hoeksema, E. Stice, E. Wade and C. Bohon, "Reciprocal relations between rumination and bulimic, substance abuse, and depressive symptoms in female adolescents”, J Abnorm Psychol, 116, pp. 198-207, 2007.

[6] M. Khosravi, "Eating disorders among patients with borderline personality disorder: understanding the prevalence and psychopathology”, J Eat Disord, 8(38), pp. 1-9, 2020.

[7] K.J. Gardner, J. Dodsworth and E.A. Selby, "Borderline personality traits, rumination and self-injurious behavior: an empirical test of the emotional cascades model in adult male offenders”, Journal of Forensic Psychology Practice, 14, pp. 398-417, 2014.

[8] M.M. Linehan, Diagnosis and treatment of mental disorders. Cognitivebehavioral treatment of borderline personality disorder. New York: Guilford Press, 1993.

[9] E.A. Selby, M.D. Anestis and T.E. Joiner, "Understanding the relationship between emotional and behavioral dysregulation: emotional cascades”, Behav Res Ther, 46, pp. 593-611, 2008.

[10] E.A. Selby, M.D. Anestis, T.W. Bender and T.E Joiner, "An exploration of the emotional cascade model in borderline personality disorder", J Abnorm Psychol, 118, pp. 375-387, 2009. 
[11] S. Nolen-Hoeksema, "Responses to depression and their effects on the duration of depressive episodes, J Abnorm Psychol, 100, pp. 569-582, 1991.

[12] E.R. Watkins, Rumination-focused cognitive-behavioral therapy for depression. New York: Guilford Press, 2016.

[13] H. Haken, Synergetics, an introduction: nonequilibrium phase transitions and self-organization in physics, chemistry and biology. New York: Springer-Verlag, 1977.

[14] E.A. Selby, A. Kranzler, E. Panza and K.B. Fehling, "Bidirectionalcompounding effects of rumination and negative emotion in predicting impulsive behavior: implications for emotional cascades”, J Pers, 84, pp. 139-153, 2016.

[15] R. Lewin, Complexity: life at the edge of chaos. Chicago: Chicago University Press, 1992.

[16] R.A. Sansone, D.A. Watts and M.W. Wiederman, "Pain and pain catastrophizing among internal medicine outpatients with borderline personality symptomatology: a cross-sectional self-report survey”, Prim Care Companion CNS Disord, 15:PCC.13m01536, 2013.

[17] C. Papageorgiou and A. Wells, Eds., Depressive rumination: nature, theory and treatment. Chichester: John Wiley\&Sons, 2004.

[18] R.A. Baer and S.E. Sauer, "Relationships between depressive rumination, anger rumination, and borderline personality features", Personality Disorders: Theory, Research, and Treatment, 2, pp. 142-150, 2011.

[19] S.E. Sauer-Zavala, P.J. Geiger and R.A. Baer, "The effect of anger rumination in the relationship between borderline personality disorder symptoms and precursors”, J Pers Disord, 27, pp. 465-472, 2013.

[20] B.T. Upton, Ruminative thought in individuals with borderline personality features, University of Kentucky Master's Theses. https://uknowledge.uky.edu/gradschool_theses/90, 2011.

[21] J.R. Peters, T.A. Eisenlohr-Moul, B.T. Upton, N.A. Talavera, J.J. Folsom and R.A. Baer, "Characteristics of repetitive thought associated with borderline personality features: a multimodal investigation of ruminative content and style”, J Psychopathol Behav Assess, 39, pp. 456-466, 2017.

[22] S. Nolen-Hoeksema, "The response styles theory", in Depressive rumination, C. Papageorgiou and A. Wells, Eds., Chichester: John Wiley\&Sons, 2004, pp. 107-124.

[23] M.J. Richman, Z. Unoka, R. Dudas and Z. Demetrovics, "Rumination in borderline personality disorder”, International Conference on Clinical Psychiatry and Psychology, 2018. https://doi.org/10.31234/osf.io/7wf4.

[24] S.E. Fahlman and C. Lebiere, "The cascade-correlation learning architecture", in Advances in neural information processing systems 2, D.S. Touretzky, Ed., Los Altos, Calif.: Morgan Kaufmann, 1990, pp.524-532.
[25] T.R. Shultz, Computational developmental psychology. Cambridge, Mass.: The MIT Press, 2003.

[26] A. Karmiloff-Smith, Beyond modularity: a developmental perspective on cognitive science. Cambridge, Mass.: The MIT Press, 1992.

[27] J.K. Brook, Cascade-correlation as a model of representational redescription, CSRP, University of Sussex, 1998.

[28] C. Pelta, "Pathological worrying and artificial neural networks", IJACSA, 11(1), 54. http://dx.doi.org/10.14569/IJACSA.2020.0110106E.

[29] C.Pelta, "Artificial neural networks for modeling the interaction between cytokines inducing lymphopenia in patients with COVID-19”,AMI,42(3 pp.157-168. https://ami.info.umfcluj.ro/index.php/AMI/article/view/786.

[30] S.E. Fahlman, "Cascade-correlation and deep learning", Carnegie Mellon University, Spring 2019. https://youtube.com.watch?v=k2mPEUZH978.

[31] E.A. Selby, S. Kondratyuk, J. Lindqvist, K. Fehling and A. Kranzler, "Temporal bayesian network modeling approach to evaluating the emotional cascade model of borderline personality disorder, Personality Disorders: Theory, Research, and Treatment, 12(1), pp. 39-50, 2021. https://doi.org/10.1037/per0000398.

[32] A.D. Redish and J.A. Gordon (Eds.), Computational psychiatry. Cambridge; Mass.: The MIT Press, 2016.

[33] D. Durstewitz and J. Seamans, "The dual-state theory of prefrontal cortex dopamine function with relevance to catechol-omethyltransferase genotypes and schizophrenia”, Biol Psychiatry 64(9), pp. 739-749, 2008.

[34] C.M. Figueroa, Reinforcement learning in individuals at risk for Alzheimer's Disease, Master's Thesis. Marquette University, 2013.

[35] R.E. Hoffman, "Computer simulations of neural information processing and the schizophrenia-mania dichotomy”, Arch Gen Psychiatry, 44(2), pp. 178-188, 1987.

[36] J.A. Reggia, E. Ruppin and R.S. Berndt (Eds.), Neural modeling of brain and cognitive disorders. Singapore: World Scientific, 1996.

[37] D.J. Stein and J. Ludik (Eds.), Neural networks and psychopathology. Cambridge: CUP, 1998.

[38] V. Mihajlovic and M. Petkovic, Dynamic bayesian networks: a state of the art. Technical Report, Computer Science Department, University of Twente, Netherlands, 2001.

[39] G. James, D. Witten, T. Hastie and R. Tibshirani, An introduction to statistical learning with applications in R. Berlin: Springer-Verlag, 2013.

[40] T. Jetter, MEMBRAIN - NN.DE. https://membrain-nn.de/main_en.htm.

[41] E.A. Selby, "Cascades of emotion: the emergence of borderline personality disorder from emotional and behavioral dysregulation”, Rev Gen Psych, 13(3):219. https://doi.org/10.1037/a0015687. 